\title{
Methylmalonic Acidemia
}

National Cancer Institute

\section{Source}

National Cancer Institute. Methylmalonic Acidemia. NCI Thesaurus. Code C98986.

A rare autosomal recessive inherited disorder caused by mutations of the MUT, MMAA, MMAB, MMADHC, and MCEE genes. It is characterized by abnormalities in the metabolism of lipids and proteins. Signs and symptoms usually appear early in life and vary from mild to life threatening. They include vomiting, dehydration, hypotonia, developmental delays, hepatomegaly, lethargy, intellectual disabilities, and chronic kidney disease. 